\title{
ANÁLISE DO ATRASO NO DIAGNÓSTICO E TRATAMENTO DO CÂNCER DE MAMA EM UM HOSPITAL PÚBLICO
}

\author{
Damila Cristina Trufelli*, Vanessa da Costa Miranda, Maria Beatriz Brisola dos Santos, Natália Moreno Perez Fraile, Priscilla Guedes Pecoroni, \\ Suzana de França Ribeiro Gonzaga, Rachel Riechelmann' Rafael Kaliks, Auro Del Giglio \\ Trabalho realizado pela Disciplina de Hematologia e Oncologia da Faculdade de Medicina do ABC e pelo Programa Integrado de Oncologia do \\ Hospital Israelita Albert Einstein, São Paulo, SP
}

\author{
* Correspondência \\ Faculdade de Medicina do ABC \\ Av. Lauro Gomes, 2000 \\ Vila Sacadura Cabral \\ Santo André/SP \\ CEP: $09060-870$
}

\section{RESUMO}

OBjetivo. Identificar as possíveis fases de atraso na condução de pacientes com câncer de mama atendidas em um hospital público, desde a suspeita até o diagnóstico e o início do tratamento.

Métodos. Estudo retrospectivo, no qual foram analisados em pacientes com câncer de mama atendidas consecutivamente no Serviço de Oncologia do Hospital Estadual Mário Covas, durante o ano de 2006, os dados relativos aos intervalos de tempo transcorrido entre a suspeita, o diagnóstico e o início do tratamento adjuvante sistêmico.

Resultados. Sessenta e oito mulheres foram incluídas. A média de idade foi de 56,3 anos (desvio padrão: 12,2 anos). Entre os intervalos analisados, o maior atraso ocorreu entre a suspeita mamográfica de câncer e a realização da biópsia (mediana de 72 dias, variação de 4 a 1095 dias), sendo este significativamente maior $(p<0,001)$ quando comparado aos demais intervalos analisados. Além disso, considerando particularmente este intervalo, verifica-se que foi significativamente maior nas pacientes com câncer de mama de estadio avançado, comparativamente àquelas de estadio inicial $(p=0,0 \mid 4)$.

Conclusão. Pacientes com câncer de mama tratadas em serviço público sofrem atrasos importantes, principalmente no período de diagnóstico de sua doença. Com base em nossos dados, otimizar o intervalo entre a mamografia e a obtenção da biópsia de lesões suspeitas parece ser a estratégia mais profícua.

UNITERMOS: Câncer de mama. Diagnóstico. Atraso. Tratamento.

\section{INTRODUÇÃO}

O câncer é uma das causas de maior mortalidade e morbidade no mundo, com mais de dez milhões de casos novos e mais de seis milhões de mortes por ano'. De todos os tipos de neoplasias, exceto o câncer de pele não-melanoma, o câncer de mama é o mais incidente nas mulheres no mundo (cerca de um milhão de casos novos estimados por ano)'. No Brasil, o câncer de mama é o mais prevalente no sexo feminino, entre 40 e 69 anos, sendo a maior causa de morte por câncer entre as mulheres". De acordo com a "Estimativa de Incidência de Câncer no Brasil para 2006", publicada pelo Instituto Nacional de Câncer (INCA), o câncer de mama foi o segundo mais incidente, com 48.930 casos, abaixo apenas do câncer de pele não-melanoma². A taxa de mortalidade relacionada ao câncer corresponde a I $5 \%$, sendo que seus índices apresentam-se crescentes e elevados, provavelmente porque a doença é diagnosticada em estadios avançados ${ }^{2}$. Informações de registros hospitalares do INCA de 2000/200 I indicam que 50\% dos tumores de mama no Brasil são diagnosticados nos estadios III e IV2. O tamanho do tumor, a presença ou ausência de receptores hormonais e a presença de linfonodos axilares e metástases são os mais consistentes indicadores prognósticos para mulheres com carcinoma mamário de estadio inicial ${ }^{3}$.

O câncer de mama pode ser detectado pelo auto-exame das mamas e/ou pela realização de mamografia e/ou ultra-sonografia, devendo ser confirmado por meio da biópsia da lesão3. A biópsia deve conter todos os elementos necessários para o adequado manejo clínico da paciente sob o ponto de vista prognóstico e terapêutico ${ }^{4}$. Com este objetivo, realiza-se na avaliação pela imunoistoquímica a pesquisa da presença de receptores hormonais (estrógeno e progesterona) e do marcador c-erb-24. O estadiamento da doença no momento da instituição do tratamento é um dos fatores mais importantes (se não o mais importante) na determinação prognóstica do paciente. Deste modo, atrasos que levem à demora diagnóstica e/ou terapêutica permitem o crescimento tumoral com potencial detrimento para as chances de cura dos pacientes.

O acesso e o tempo para o diagnóstico e tratamento do câncer de mama variam nas diversas regiões do país, dependendo de fatores geográficos e socioeconômicos. Estudo preliminar, realizado nos três serviços de oncologia clínica ligados à Faculdade de Medicina do ABC, em Santo André, mostrou que o atraso no diagnóstico do câncer de mama está, em grande parte, relacionado ao tempo que a paciente demora a procurar os serviços de saúde a partir da detecção do primeiro sintoma ou sinal (por exemplo, a palpação de nódulo ou outra alteração na mama) $)^{5}$. A partir do primeiro sintoma, a mediana de tempo para agendar a 
primeira consulta foi de três meses (0-58,2 meses). Secundariamente, $\mathrm{o}$ atraso foi devido à demora entre a primeira consulta e a realização da biópsia. A demora entre o dia da primeira consulta até $\mathrm{o}$ ato da biópsia foi de dois meses (0-49 meses) $)^{5}$. Dados de um estudo do Instituto Nacional do Câncer dos Estados Unidos $(\mathrm{NCl})$ indicam que aproximadamente $40 \%$ das mulheres relataram um atraso no diagnóstico do câncer de mama de quatro semanas ou mais entre o primeiro sintoma e o resultado do anatomopatológico, e outras 25\% queixaram-se de um retardo maior que oito semanas 6 . A maioria delas atribuiu o fato a deficiências no sistema de saúde, no qual a média de atraso foi de II a 13 semanas $^{6}$. O atraso no tratamento do câncer de mama pode ser dividido em três fases. A primeira ocorre a partir do primeiro sintoma até a consulta médica. A segunda ocorre da primeira consulta até o acesso ao serviço de referência especializado em seu tratamento, e a terceira, da primeira avaliação neste serviço até o início do tratamento específico ${ }^{7}$.

Estudos têm evidenciado que o diagnóstico e o tratamento precoce do câncer de mama podem reduzir mortalidade específica ${ }^{6,8}$. Da mesma forma, em uma revisão sistemática, atrasos de três a seis meses entre o sintoma inicial e o tratamento adjuvante foram claramente associados com a diminuição da taxa de sobrevivência destas pacientes ${ }^{9}$. Além disso, Olivotto et al. sugerem que atrasos de seis a 12 meses no diagnóstico de câncer de mama assintomático estão associados com a progressão da doença, determinados pelo aumento do risco de metástase linfonodal e maior tamanho do tumor. ${ }^{10}$ No entanto, há estudos que observaram que atrasos maiores que 60 dias entre a detecção do primeiro sintoma pelo médico e o diagnóstico definitivo não afetam o prognóstico da paciente com câncer de mama ${ }^{11-14}$.

Assim, o objetivo do presente estudo foi identificar as possíveis fases de atraso na condução das pacientes dentro do serviço de oncologia de um hospital público, o Hospital Estadual Mário Covas. Especificamente, enfocaremos os atrasos que ocorreram com a paciente já inserida no fluxo de atendimento deste serviço. Visamos assim, identificar possíveis falhas resultando em atrasos de atendimento dentro de um serviço público. A partir destes resultados poderá ser realizado um plano de ação para minimizar os períodos de atraso neste serviço.

\section{Métodos}

Este é um estudo retrospectivo, aprovado pelo Comitê de Ética e Pesquisa da Faculdade de Medicina do ABC. Os dados do estudo foram extraídos do registro administrativo de datas e prontuários médicos. Pacientes consecutivas com diagnóstico de câncer de mama e que foram atendidas em primeira consulta em um ambulatório do serviço de Oncologia do Hospital Estadual Mário Covas durante o ano de 2006 foram consideradas elegíveis. Foram excluídas pacientes para as quais não haviam informações essenciais nos prontuários referentes aos intervalos a serem descritos adiante, e aquelas que realizaram seus exames fora do sistema público, por possuírem acesso a planos de assistência privada. Os seguintes intervalos foram analisados: I) entre a mamografia inicial e a realização da biópsia; 2) entre a realização da biópsia e seu resultado (laudo da anatomia patológica); 3) entre o resultado anatomopatológico da biópsia e o resultado da imunoistoquímica; 4) entre o resultado final da biópsia e a cirurgia; 5) entre o resultado anatomopatológico da biópsia e o início do tratamento quimioterápico neo-adjuvante (quando aplicável); 6) entre o final do tratamento quimioterápico neo-adjuvante e a cirurgia; 7) entre a cirurgia e o resultado do estadiamento anatomopatológico e 8) entre o estadiamento anatomopatológico e o início do tratamento adjuvante sistêmico.

Para a análise estatística, utilizou-se o programa SPSS ${ }^{\circledR}$ versão 13.0 (SPSS ${ }^{\circledR}$ Inc; llinois, USA). A escolha das medidas de tendência central e dispersão dos valores que compõem as amostras, assim como dos testes estatísticos para comparação entre as mesmas, baseou-se nos tipos de distribuição, segundo o teste de Kolmogorov-Smirnov. Os valores obtidos pelo estudo de cada variável quantitativa foram organizados e descritos por meio da mediana, da média e do desvio padrão. Para as qualitativas, utilizamos frequêencias absolutas e relativas. Comparações da freqüência de um fenômeno entre grupos de variáveis qualitativas foram realizadas com aplicação do teste exato de Fisher ou teste do Qui-quadrado. Para a comparação entre variáveis quantitativas e qualitativas, utilizamos os testes " $t$ " de Student para duas populações amostrais, e a análise de variância (ANOVA) com teste auxiliar de Bonferroni, quando aplicável, para três ou mais populações. Para todos os testes empregados, adotou-se o valor de significância estatística menor ou igual a $5 \%(p=0,05)$.

\section{Resultados}

No presente estudo foram analisados os prontuários de 73 pacientes do sexo feminino, com média de idade de 56,34 \pm 12, 198 anos, diagnosticadas com câncer de mama e que realizaram tratamento oncológico no Hospital Estadual Mário Covas. Foram excluídas cinco pacientes: três por não constarem dados essenciais no prontuário, como exemplo as datas das respectivas etapas entre 0 diagnóstico e tratamento, e duas por terem feito todos os exames diagnósticos em serviço privado. Das 68 pacientes incluídas para a análise final, 83, $1 \%$ realizaram a mamografia como exame inicial e $42,4 \%$ se encontravam no estadio II da doença. Em relação ao diagnóstico imunoistoquímico, 53,3\% das mulheres apresentavam tumores com positividade para receptores de estrógeno e progesterona; $24,6 \%$ apresentavam positividade para c-erb-B2. Do total das pacientes que passaram por intervenção cirúrgica (56 pacientes), 53,6\% realizaram mastectomia. Apenas 20 pacientes $(29,9 \%)$ realizaram tratamento neo-adjuvante. Quarenta e nove mulheres (72\%) realizaram tratamento adjuvante, entre elas, $28,6 \%$ foram tratadas apenas com quimioterapia e $22,4 \%$ receberam quimioterapia, radioterapia e hormonioterapia. Quatro mulheres (5,9\%) evoluíram a óbito, devido às complicações da própria doença. As demais características estão descritas na Tabela I.

A partir da análise estatística, foi observado que o maior intervalo no diagnóstico do câncer de mama ocorreu entre a mamografia e a biópsia, sendo este significativamente maior quando comparado a outros intervalos (ANOVA, $p<0,00 \mathrm{I}$ ). As medianas de tempo dos intervalos estão descritas na Tabela 2. 


\begin{tabular}{|c|c|}
\hline Características & $\mathbf{N}(\%)$ \\
\hline $\begin{array}{l}\text { Média de idade Mamografia } \\
\text { Sim } \\
\text { Não }\end{array}$ & $\begin{array}{c}56,34 \text { anos } \pm 12,198 \\
54(83,1) \\
1 \mid(16,9)\end{array}$ \\
\hline Receptor hormonal & \\
\hline $\begin{array}{l}\text { Estrógeno - / Progesterona - } \\
\text { Estrógeno + / Progesterona - } \\
\text { Estrógeno - Progesterona + } \\
\text { Estrógeno + / Progesterona + } \\
\text { c-erb-2 }\end{array}$ & $\begin{array}{c}16(26,7) \\
7(11,7) \\
5(8,3) \\
32(53,3)\end{array}$ \\
\hline $\begin{array}{l}\text { Negativo } \\
\text { Positivo }\end{array}$ & $\begin{array}{l}43(75,4) \\
14(24,6)\end{array}$ \\
\hline Estadiamento & \\
\hline $\begin{array}{l}\text { Estadio I } \\
\text { Estadio II } \\
\text { Estadio III } \\
\text { Estadio IV }\end{array}$ & $\begin{array}{c}17(25,7) \\
28(42,4) \\
17(25,8) \\
4(6,1)\end{array}$ \\
\hline Tipo de cirurgia & \\
\hline $\begin{array}{l}\text { Setorectomia } \\
\text { Quadrantectomia } \\
\text { Mastectomia }\end{array}$ & $\begin{array}{c}2(3,6) \\
24(42,9) \\
30(53,6)\end{array}$ \\
\hline Tratamento neo-adjuvante & \\
\hline $\begin{array}{l}\text { Sim } \\
\text { Não }\end{array}$ & $\begin{array}{l}20(29,9) \\
47(70,1)\end{array}$ \\
\hline Tratamento adjuvante & \\
\hline $\begin{array}{l}\text { Quimioterapia (QT) } \\
\text { Radioterapia (RT) } \\
\text { Hormonioterapia }(H T) \\
\text { QT + RT } \\
\text { QT + HT } \\
\text { RT + HT } \\
\text { QT + RT + HT }\end{array}$ & $\begin{array}{l}14(28,6) \\
4(8,2) \\
8(16,3) \\
2(4,1) \\
2(4,1) \\
8(16,3) \\
11(22,4)\end{array}$ \\
\hline $\begin{array}{l}\text { Óbito } \\
\text { Não } \\
\text { Sim }\end{array}$ & $\begin{array}{c}64(94,1) \\
4(5,9)\end{array}$ \\
\hline
\end{tabular}

*NOTA: 0 total de pacientes avaliadas para cada variável é inferior a 68, pois para algumas destas características não havia informaçōes no prontuário.

Tabela 2 - Medianas de tempo dos intervalos entre as etapas estudadas

Intervalos Mediana (min-máx)

(dias)

Mamografia - Biópsia

Biópsia - Resultado da biópsia

$72(4-1095)$

I $(0-68)^{*}$

Resultado da biópsia - Imunoistoquímica

$14,5(0-299)$

Resultado da biópsia -

Tratamento neo-adjuvante

Tratamento neo-adjuvante - Cirurgia

$14,5(0-164)$

$55,5(0-120)$

$36(0-316)$

$13(0-98)$

Cirurgia - Anatomopatológico

$35(13-627)$

$189(5-1178)$

Anatomopatológico - Tratamento adjuvante

Mamografia - Tratamento adjuvante

*Os dados referentes ao intervalo entre a entrada da biópsia no serviço de anatomia patológica e seu resultado foram retirados dos laudos anatomopatológicos.

\begin{tabular}{lcc}
\hline \multicolumn{3}{c}{$\begin{array}{c}\text { Tabela 3 } \\
\text { a biópsia de acordo como o estadio da doença }\end{array}$} \\
\hline $\mathbf{N}(\%)$ & Estadios & $\begin{array}{c}\text { Tempo (dias) } \\
\text { Mediana (min-máx) }\end{array}$ \\
\hline $17(25,7 \%)$ & Estadio I & $84(4-309)$ \\
$28(42,4 \%)$ & Estadio II & $59,5(4-22$ I) \\
$17(25,7 \%)$ & Estadio II & $73(42-1095)$ \\
$4(6,2 \%)$ & Estadio IV & $504,5($ ( I $6-893)$ \\
\hline$* N=$ número de pacientes em cada estadio &
\end{tabular}

A análise estatística também mostrou que o estadio IV da doença está relacionado a um maior intervalo entre a mamografia e a biópsia (ANOVA, $p=0,0 \mid 4$ ). (Tabela 3).

Vale ressaltar que não foram encontradas associações estatisticamente significantes em nenhum dos intervalos de tempo entre as sucessivas etapas de diagnóstico e tratamento para as variáveis: idade, positividade para receptores hormonais e c-erb2, diagnóstico por mamografia, tipo de cirurgia e de tratamento adjuvante e óbito.

\section{Discussão}

Nosso estudo mostra que o maior atraso na condução dos casos de câncer de mama ocorreu entre a mamografia e a realização da biópsia da lesão suspeita. Ele também sugere que a maior demora no diagnóstico está relacionada a estadios mais avançados da doença. Em nosso serviço, isto não se deveu a um viés de seleção, já que a investigação do estadiamento por exames radiológicos sucedeu a realização da biópsia. Chama a atenção, o intervalo entre a mamografia suspeita e o início da terapia sistêmica (> I 80 dias), já que este é o intervalo de tempo no qual potenciais micrometástases podem se implantar, tornando a doença metastática e geralmente incurável.

Estudos americanos comparando a etnia das pacientes com o câncer de mama e, conseqüentemente, o seu nível socioeconômico, mostraram que aquelas de raça negra apresentaram maiores atrasos tanto no diagnóstico quanto no tratamento da doença, quando comparadas àquelas de raça branca ${ }^{15,16}$. Possíveis explicações dadas a este fato foram: ausência de conhecimento a respeito da gravidade do quadro e a importância de se fazer um diagnóstico precoce do câncer de mama e a tendência ao pensamento de fatalidade diante da doença ${ }^{15,16}$. Essas mesmas explicações poderiam ser empregadas para as pacientes atendidas em hospitais públicos brasileiros.

A detecção e o início precoce do tratamento do câncer estão relacionados à maior taxa de cura das pacientes com câncer de mama precoce. ${ }^{17}$ Quanto mais rápido for a instituição do tratamento do câncer para tumores iniciais (não-metastáticos), maior é a chance da cirurgia ser curativa e de não ocorrer disseminação da doença $a^{17,18}$. Estudos demonstram que o impacto na sobrevida de pacientes com neoplasia mamária está associado à relação entre o atraso no diagnóstico, tratamento e o estadiamento do câncer ${ }^{19-22}$. Richards et al. ${ }^{17}$ observaram em uma metaanálise que o atraso no diagnóstico está associado com estágios mais avançados da doença, enquanto que Olivotto et al. demonstraram que este atraso está relacionado ao maior risco de metástase linfonodal e maior tamanho tumoral, 
determinantes do estadiamento ${ }^{10}$. Da mesma forma, o presente estudo evidenciou uma relação significante entre o maior intervalo da mamografia à biópsia, e o estadio IV à época da cirurgia. Contudo, havia apenas quatro pacientes com estádio IV e esta associação pode ter ocorrido por chance.

No entanto, a literatura não se mostra homogênea no que diz respeito à relação entre menores intervalos no diagnóstico e tratamento do câncer de mama e sua influência no prognóstico destas pacientes. Em um estudo de Comber et al. ${ }^{14}$, realizado na Irlanda, comparando diversos tipos de tumores, foi observado que os menores atrasos eram atribuídos ao câncer de mama e que este tempo não estava associado ao pior prognóstico ou menor sobrevida. Além disso, outros autores afirmam que apesar do atraso no diagnóstico do câncer de mama ser responsável por causar ansiedade, dentre outros distúrbios psicológicos nas pacientes com a doença, este não têm efeito sobre o prognóstico ${ }^{11-13}$.

Embora os intervalos de atraso entre a biópsia e o resultado do anatomopatológico da biópsia e da imunoistoquímica ainda não sejam ideais, eles não parecem ser significativos em relação ao atraso total para o tratamento definitivo. O resultado da imunoistoquímica, apesar de extremamente demorado para padrões de excelência, acaba sendo rápido o suficiente para que cada paciente que precisa de hormonioterapia adjuvante consiga iniciar este tratamento no tempo devido. O intervalo entre o fim da quimioterapia neoadjuvante e a cirurgia é extremamente longo e inadequado do ponto de vista oncológico, pois permite a progressão de doença entre a resposta máxima ao tratamento e o procedimento cirúrgico. 0 intervalo entre o estadiamento baseado no anatomopatológico (estadiamento patológico) e o início do tratamento adjuvante é longo e pode, teoricamente, comprometer o prognóstico oncológico do paciente, embora seja impossível prever quanto do benefício da adjuvância é perdido em função de cada mês de atraso em seu início. A maioria dos estudos clínicos de tratamento adjuvante em oncologia utiliza a marca dos "três meses" como um ponto de corte para a inclusão de pacientes. Desta forma, os benefícios da terapia adjuvante relatados na literatura limitam-se a pacientes que receberam o tratamento até três meses após o diagnóstico patológico. Vale ressaltar que este é um ponto de corte arbitrário e o intervalo ideal para se iniciar a terapia adjuvante é desconhecido. Vários estudos mostraram resultados controversos quanto ao impacto do intervalo entre a cirurgia e a instituição do tratamento na sobrevida global dos doentes com câncer ${ }^{23,24}$. Do ponto de vista de importância clínica dos diferentes atrasos, os três intervalos mais importantes, em ordem decrescente de gravidade, são: mamografia - biópsia, resultado da biópsia - cirurgia, resultado do exame anatomopatológico tratamento adjuvante.

Baseados nestes resultados, consideramos urgente a instituição de estratégias para corrigir os atrasos, nesta ordem de prioridade. Acreditamos que uma assistente social ou enfermeira dedicada ao estabelecimento do fluxo de pacientes com câncer de mama, e que tenha autorização para fazer agendamentos ou encaminhar pacientes para outros serviços, dependendo da urgência do caso, solucionaria, ao menos parcialmente, estes atrasos e beneficiaria sobremaneira o atendimento global às pacientes com câncer de mama na nossa instituição. Por exemplo, uma dada paciente agendada para mamografia seria convocada por esta assistente social ou enfermeira se o resultado requisesse uma biópsia. A partir deste momento, os agendamentos para realização da biópsia, obtenção do seu resultado, agendamento eficiente com o médico (oncologista e/ou mastologista) que deverá explicar à paciente o resultado e delinear sua conduta, assim como as demais consultas até o início do tratamento, estariam sob sua responsabilidade. Portanto, esta função de coordenação do fluxo de pacientes que necessitem de biópsia mamária seria, a nosso ver, a principal medida para evitar perdas de seguimento e atrasos indevidos no atendimento destas pacientes.

\section{Conclusão}

As pacientes com diagnóstico de câncer de mama sofrem atrasos em várias etapas do seu diagnóstico e tratamento oncológico. Em nosso meio, o maior atraso consiste no intervalo entre a mamografia e a biópsia. A nosso ver, uma coordenadora do fluxo de pacientes com necessidade de biópsia mamária a partir de uma mamografia suspeita poderia diminuir os atrasos que hoje se observam no cuidado destas pacientes. Prover mamografia para mulheres da população geral é de suma importância, porém é imperativo que o sistema tenha meios e organização para atuar sobre as anormalidades encontradas neste exame. Acreditamos que assim reduziremos a progressão da neoplasia para estadios mais avançados e incuráveis.

\section{Conflito de interesse: não há}

\section{SUMMARY \\ ANALYSIS OF DELAYS IN DIAGNOSIS AND TREATMENT OF BREAST CANCER PATIENTS AT A PUBLIC HOSPITAL}

BACKGROUND. To identify potential delays in the management of patients with breast cancer examined at a public hospital, from time of suspicion until diagnosis and beginning of treatment.

METHODS. Retrospective study which analyzed data related to time elapsed in different intervals between suspicion, diagnosis and onset of systemic cancer-directed treatments, of consecutive breast cancer patients cared for at the medical oncology clinic of the Mario Covas Hospital during 2006.

RESULTS. Sixty-eight women, with a mean age of 56.3 years (standard deviation: 12.2 years), were included. Ofall the intervals, the longest delay occurred between the mammographic suspicion of cancerand performance of biopsy (median of 72 days, range: 4 - 1095 days); this was significantly longer $(P<0.001)$ than in the other intervals analyzed. Furthermore, this interval in particular, was significantly longer in patients with advanced stage breast cancer when compared to those at the initial stage $(P=0.014)$.

CONCLUSION. Breast cancer patients treated in a public hospital in Brazil suffer delays, especially during the diagnosis of their disease. Minimizing the time between mammography and biopsy of suspicious lesions appears to be the most crintical step to correct this situation. [Rev Assoc Med Bras 2008; 54(I): 72-6]

KEY WORDS: Breast cancer. Diagnosis. Delays. Treatment. 


\section{REFERÊNCIAS}

I. Surveillance epidemiology and end results (SEER). National Cancer Institute. [cited 2007 mayo 22]. Disponível em: http://seer.cancer.gov.

2. Estimativas da incidência e mortalidade por câncer no Brasil. Ministério da Saúde: Instituto Nacional do Câncer. [citado 6 maio 2007]. Disponível em: http://www.inca.gov.br.

3. Câncer de mama. Ministério da Saúde: Instituto Nacional do Câncer. citado 6 maio 2007]. Disponível em: http://www.inca.gov.br.

4. Barros ACSD, Barbosa EM, Gebrim LH. Projeto diretrizes: diagnóstico e tratamento do câncer de mama. Sociedade Brasileira de Mastologia. [citado 6 maio 2007]. Disponível em: http:// www.sbmastologia.com.br.

5. Trufelli DC, Bensi CG, Pane CEV, Ramos E, Otsuka FC, Tannous NG, et al. Onde está o atraso? Avaliação do tempo necessário para o diagnóstico e tratamento do câncer de mama nos serviços de oncologia da Faculdade de Medicina do ABC. Rev Bras Mastol. 2007; 17(I): | 4-8.

6. Caplan LS, Edwards BK, Helzlsouer KJ, Shapiro S, Wesley MN. Reasons for delay in breast cancer diagnosis. Prev Med. 1996;25(2):21 8-24.

7. Coates AS. Breast cancer: delays, dilemmas, and delusions. Lancet 1999;353(9|58): I | I2-3

8. Nystrom L, Rutqvist LE, Wall L. Breast cancer screening with mammography: overview of the Swedish randomized trials. Lancet 1993;34 I(885 I):973-8.

9. Ramirez AJ, Westcombe AM, Burgess CC. Factors predicting delayed presentation of symptomatic breast cancer: a systematic review. Lancet. | 999;353(9|59): | |27-3|.

10. Olivotto IA, Gomi A, Bancej C, Brisson J, Tonita J, Kan L, et al. Influence of delay to diagnosis on prognostic indicators of screen-detected breast carcinoma. Cancer. 2002;94(8):2। 43-50.

II. Dennis CR, Gardner B, Lim B. Analysis of survival and recurrence vs. patient and doctor delay in treatment of breast cancer. Cancer. 1975; 35(3):7| 4-20.

12. Afzelius $P$, Zedeler $K$, Sommer $H$, Mouridsen $H T$, Blichert-Toft $M$. Patient's and doctor's delay in primary breast cancer. Prognostic implications. Acta Oncol. 1994;33(4):345-5I.

13. Tartter PI, Pace D, Frost M, Bernstein JL. Delay in diagnosis of breast cancer. Ann Surg. 1999;229(1):91-6.
14. Comber H, Cronin DP, Deady S, Lorcain PO, Riordan P. Delays in treatment in the cancer services: impact on cancer stage and survival. Ir Med J. 2005;98(8):238-9.

15. Gullatte MM, Phillips JM, Gibson LM. Factors associated with delays in screening of self-detected breast changes in African-American women. J Natl Black Nurses Assoc. 2006; 17(1):45-50.

16. Gorin SS, Heck JE, Cheng B, Smith SJ. Delays in breast cancer diagnosis and treatment by racial/ethnic group. Arch Intern Med. 2006; 1 66(20):2244-52.

17. Richards MA, Westcombe AM, Love SB, Littlejohns P, Ramirez AJ. Influence of delay on survival in patients with breast cancer: a systematic review. Lancet. 1999;353(9 I59): I I 19-26.

18. Montella M, Crispo A, D'Aiuto G, De Marco M, Bellis G, Fabbrocini G, et al. Determinant factors for diagnostic delay in operable breast cancer patients. Eur J Cancer Prev. 200 I; I 0(1):53-9.

19. Sainsbury R, Haward B, Rider L, Johnston C, Round C. Influence of clinician workload and patterns of treatment on survival from breast cancer. Lancet. 1995;345(8960): 1 265-70.

20. Richardson JBL, Bernstein C, Burciaga C, Danley R, Ross R. Stage and delay in breast cancer diagnosis by race, socioeconomic status, age and year. Br J Cancer. 1992;65(6):922-6.

21. Montella M, Crispo A, Botti G, De Marco M, de Bellis G, Fabbrocini G, et al. An assessment of delays in obtaining definitive breast cancer treatment in Southern Italy. Br Cancer Res Treat. 200 I;66(3):209- 15.

22. Cant PJ, Yu DSL. Impact of the ' 2 week wait' directive for suspected cancer on service provision in a symptomatic breast clinic. $\mathrm{Br} J$ Surg. 2000;87(8): 1082-6.

23. Cold S, Düring M, Ewertz M, Knoop A, Møller S. Does timing of adjuvant chemotherapy influence the prognosis after early breast cancer? Results of the Danish Breast Cancer Cooperative Group (DBCG). Br J Cancer. 2006;94(6):938-9.

24. Shannon C, Ashley S, Smith IE. Does timing of adjuvant chemotherapy for early breast cancer influence survival? J Clin Oncol. 2003;2I (20):3792-7.

Artigo recebido: 26/07/07

Aceito para publicação: 18/09/07 